\title{
The London 2012 Olympics - will there be a legacy for mental health?
}

\author{
Alan Currie ${ }^{1}$ \\ The Psychiatrist (2012), 36, 281-283, doi: 10.1192/pb.bp.111.037895 \\ ${ }^{1}$ Northumberland, Tyne and Wear NHS \\ Foundation Trust, UK \\ Correspondence to Alan Currie \\ (alan.currie@ntw.nhs.uk) \\ First received 19 Nov 2011, final \\ revision 11 Feb 2012, accepted 2 Mar \\ 2012 \\ Summary The host country's legacy after the Olympic Games is multifaceted. \\ Alongside such diverse elements as tourism, commerce and transport sit the health \\ benefits of increased participation in sports and provision of the highest quality \\ medical support for the nation's elite sporting performers. Mental health, however, \\ merits no specific mention. This could be a missed opportunity to create a legacy that \\ promotes the mental health benefits of exercise as well as ensuring that the mental \\ health needs of elite sportsmen and women are recognised and met in the same \\ manner as their physical health needs.
}

Declaration of interest None.
One justification for the expenditure incurred by the host country of a major sporting event is the legacy that remains when the competitions are over. For the 2012 London Olympics the hope is for a legacy that encompasses urban regeneration, sports facilities, commercial opportunities, a boost to tourism, sporting success for the nation and an opportunity to showcase the country as a place to live, to visit and in which to do business. ${ }^{1}$ At the head of the list of legacy promises is a pledge to make the UK a world-leading sporting nation and this is accompanied by a government statement that 'we plan to use the Games to boost sport and physical activity' (p. 2). ${ }^{1}$ Items that relate specifically to health and well-being are contained within this pledge. They are the aspiration to encourage people, and especially young people, to become more physically active alongside improved medical care for the country's elite performers. The Paralympic Games are also promoted as producing a legacy of increased sports participation and associated improvements in the quality of life of people with disabilities. $^{2}$

Mental health does not specifically feature in any aspects of the health legacy. For example, the perceived health benefits of increased exercise participation are primarily in the physical health realm and mental health benefits are not specifically targeted. The disability legacy aims to use the Paralympic Games to challenge unhelpful stereotypes and stigma, but by focusing on the physical impairments on which qualification for the Paralympics is dependent there is no room to include mental illness-related disability. What then could be the mental health legacy of the 2012 London Games?

\section{Exercise as a health intervention}

The mental health benefits of participation in physical activity may seem self-evident, although they are disappointingly hard to prove. In young people small but non-significant improvements have been noted in mental health, ${ }^{3}$ specifically in anxiety and depression ${ }^{4}$ and self-esteem, ${ }^{5}$ and there is a consistent association between sedentary time spent using screen-based media and poorer mental health. ${ }^{3}$ A modest and not statistically significant clinical improvement is seen when adult patient populations are studied, including in conditions such as depression ${ }^{6}$ and schizophrenia. ${ }^{7}$ Most studies describe a heterogeneous mix of exercise interventions and although this reflects real life, where opportunities for sports participation thankfully come in great variety, it does reduce the likelihood of a robustly positive finding. However, in contrast to other interventions including psychotherapies, there is a paucity of any demonstrable negative effects of exercise participation.

Health is not the only reason for engaging in physical activity and many participants cite the opportunities created for social interaction as an important factor. ${ }^{8}$ This may be especially important for those who are regularly and frequently excluded from a participative life by reason of their illness or condition. Those with enduring mental illnesses are frequently multiply disadvantaged in this way and find themselves excluded from opportunities in education, employment and housing as well as leisure activities. $^{9}$ Participation in leisure activities among people with schizophrenia is especially low. ${ }^{10}$ Might sport be a vehicle where the journey to participation, inclusion and recovery begins? There are already many, although often isolated, examples of sport and exercise being used to promote social inclusion for psychiatric patients with the greatest disability and disadvantage (www.pmasports.com). There will be potential advantages for mental health services in delivering socially inclusive outcomes and in promoting recovery if they are able to form and sustain links with sports organisations. The psychiatrists who embrace this will be those who build an Olympic legacy for mental health. 
Exercise as an intervention to enhance the mental well-being of the population falls clearly within the remit of a pledge to improve health via participation in sport, whereas using sport as a driver of social inclusion has a direct parallel with the Paralympics pledge to enhance the lives of those who live with disability and disadvantage. It is disappointing that their inclusion in the Olympic legacy statement is not more explicit.

\section{Mental illness and athletes}

Sport and exercise medicine is a multidisciplinary field that focuses on two areas. First, the use of exercise as an intervention to improve outcomes across a range of health-related conditions including obesity and cardiac disease, and second, the provision of specialist treatment for medical problems related to sport. The latter is closely related to another facet of the Olympic legacy of ensuring the best available sports medicine support for the country's leading performers. If there is to be an Olympic legacy for mental health, this could and should include provision of psychiatric services to athletes.

The prevalence of psychiatric morbidity in elite sport is not insignificant. Perhaps the most frequently studied is the high prevalence of eating disorders especially, but not exclusively, in sports such as gymnastics, endurance running and weight category sports, for example judo, boxing and wrestling. ${ }^{11}$ Alongside this is an increasing awareness of the sports-specific factors that contribute to an increased risk of developing a psychiatric disorder. ${ }^{12}$ Depressive disorders are at least as common as in non-sporting populations ${ }^{13}$ and again there has been interest in how the sports environment contributes to this. ${ }^{14,15}$ Public interest in such cases in elite sport may be helpful in raising concerns and has been a stimulus to increased awareness and further research in, for example, Germany ${ }^{16}$ and the USA. ${ }^{17}$

To address conspicuous psychiatric morbidity in the sports arena there has to be acknowledgement within sport that a problem needs addressing and expertise from psychiatry that is willing and able to assist. It is perhaps no coincidence that eating disorders, the sports-related psychiatric condition about which there is greatest awareness, is the one area where the UK has both a national sports-specific guideline ${ }^{18}$ and a national treatment and research centre (opened in Loughborough in 2011). However, whereas most sports teams would expect to know, for example, how to access orthopaedic care and even to have a close and ongoing working relationship with an orthopaedic specialist, could they say the same about their relationship with mental health services and psychiatrists? Such relationships exist (baseball in the USA is a worthy exception) but they are not the norm. ${ }^{19}$ Contrast the rapid, multidisciplinary response of a team of experts to a physically injured sportsman with the typical response when mental health problems are (often all too reluctantly) disclosed. As advocates for our patients it is our duty to speak out where discrimination and stigma exist and to appeal for equality of access to treatments for physical and mental health problems. Better medical care for elite sports performers, an Olympic legacy pledge, has to include ready access to psychiatric expertise. It would be a major contribution to the de-stigmatisation of mental illness if the psychiatric care of Team GB were accorded parity with other healthcare needs and with it recognition that even for the nation's elite sportsmen and women there is no health without mental health. That really would be an Olympian legacy.

\section{Are psychiatrists prepared?}

Do psychiatrists need any special skills to treat sportsmen and women? As clinicians we must understand not only a syndrome or illness but also the context in which this has occurred - in this case, sport. This means exploring the hopes, aspirations and fears of the individual, what they value most and what they might lose, the key individuals and relationships is their life, the external stressors and supports to which they are exposed and much else besides. We do this for all our patients and a willingness to understand and work with the context in which a disorder has arisen is one of our key competencies. ${ }^{20}$ We may not know it but we already have the skills to assess and treat special people (sportsmen and women) in the unusual environment of elite sport.

The necessary knowledge need not extend to a detailed understanding of a particular sport and its rules. Indeed, a psychiatrist who is too close to a sport and its leading performers may experience a difficult countertransference that impedes his or her ability to provide the highest quality treatment. $^{21}$ The essential competencies are those of any good psychiatrist. Although interviewing a sportsperson may require flexibility and a willingness to consider diverse and 'athlete-centred' venues for the consultation, this is no different to the approach of any modern community psychiatrist. Athletes may be a hard-to-reach group but they are deserving of our attention and we have the skills to assist them. Making our expertise available is our contribution to the Olympic legacy.

\section{About the author}

Alan Currie is a consultant psychiatrist with Northumberland, Tyne and Wear NHS Foundation Trust and an honorary clinical lecturer at the University of Newcastle.

\section{References}

1 Department for Culture, Media and Sport. Before, During and After: Making the Most of the London 2012 Games. DCMS, 2008.

2 Department for Culture, Media and Sport. London 2012: A Legacy for Disabled People. Setting New Standards, Changing Perceptions. DCMS, 2010.

3 Biddle SJH, Asare M. Physical activity and mental health in children and adolescents: a review of reviews. Br J Sports Med 2011; 45: 886-95.

4 Larun L, Nordheim LV, Ekeland E, Hagen KB, Heian F. Exercise in prevention and treatment of anxiety and depression among children and young people. Cochrane Database Syst Rev 2006; 3: CD004691.

5 Ekeland E, Heian F, Hagen KB, Abbott JM, Nordheim L. Exercise to improve self-esteem in children and young people. Cochrane Database Syst Rev 2004; 1: CD003683.

6 Mead GE, Morley W, Campbell P, Greig CA, McMurdo M, Lawlor DA Exercise for depression. Cochrane Database Syst Rev 2009; 3: CD004366. 
7 Gorczynski P, Faulkner G. Exercise therapy for schizophrenia. Cochrane Database Syst Rev 2010; 5: CD004412.

8 Foster C, Hillsdon M, Cavill N, Allender S, Cowburn G. Understanding Participation in Sport: A Systematic Review - September 2005. Sport England, 2005

9 Boardman J. How are people with mental health problems excluded? In Social Inclusion and Mental Health (eds J Boardman, A Currie, H Killaspy, G Mezey): 135-72. RCPsych Publications, 2010.

10 Shimitras L, Fossey E, Harvey C. Time use of people living with schizophrenia in north London catchment area. Br J Occup Ther 2003; 66: 46-54.

11 Sundgot-Borgen J, Torstveit MK. Prevalence of eating disorders in elite athletes is higher than in the general population. Clin J Sports Med 2004 14: $25-32$

12 Thompson RA, Sherman RT. Risk factors for the development of problem eating. In Eating Disorders in Sport (eds RA Thompson, RT Sherman): 57-80. Routledge, 2010.

13 Yang J, Peek-Asa C, Corlette JD, Cheng G, Foster DT, Albright J. Prevalence of and risk factors associated with symptoms of depression in competitive collegiate student athletes. Clin J Sport Med 2007; 17: $481-7$
14 Burton RW. Mental illness in athletes. In Sport Psychiatry (eds D Begel, RW Burton): 61-81. WW Norton, 2000.

15 Mummery K. Depression in sport. Lancet 2005; 366: s36-7.

16 Markser VZ. Sport psychiatry and psychotherapy. Mental strains and disorders in professional sports. Challenge and answer to societal changes. Eur Arch Psych Clin Neurosci 2011; 261 (suppl 2): s182-5.

17 Guskiewicz KM, Marshall SW, Bailes J, McCrea M, Harding PH, Matthews $A$, et al. Recurrent concussion and risk of depression in retired football players. Med Sci Sports Exerc 2007; 39: 903-9.

18 UK Sport. Eating Disorders in Sport: A Guideline Framework for Practitioners Working with High Performance Athletes. UK Sport, 2007 (http://www.uksport.gov.uk/publications/eating-disorders-in-sport).

19 Torre PS. A light in the darkness. Sports Illustrated 2010; 21 June.

20 Royal College of Psychiatrists. Role and Responsibilities of the Consultant in General Adult Psychiatry (Council Report CR140). Royal College of Psychiatrists, 2006

21 Begel D. An overview of sport psychiatry. Am J Psychiatry 1992; 149 606-14. 